Acta Crystallographica Section E

Structure Reports

Online

ISSN 1600-5368

Iain D. H. Oswald, ${ }^{a} *$ W. D. Sam Motherwell $^{b}$ and Simon Parsons ${ }^{a}$

${ }^{\text {a }}$ School of Chemistry, The University of Edinburgh, King's Buildings, West Mains Road, Edinburgh EH9 3JJ, Scotland, and bambridge Crystallographic Data Centre, 12 Union Road, Cambridge CB2 1EZ, England

Correspondence e-mail: iain.oswald@ed.ac.uk

\section{Key indicators}

Single-crystal X-ray study

$T=150 \mathrm{~K}$

Mean $\sigma(\mathrm{C}-\mathrm{C})=0.002 \AA$

$R$ factor $=0.048$

$w R$ factor $=0.120$

Data-to-parameter ratio $=16.7$

For details of how these key indicators were automatically derived from the article, see http://journals.iucr.org/e.

\title{
A 1:1 co-crystal of quinol and pyridine
}

A co-crystal of quinol and pyridine would be expected to form with 1:2 stoichiometry because quinol has two hydrogen-bond donors and pyridine has one hydrogen-bond acceptor which is more basic than phenolic oxygen. We report the structure of a 1:1 co-crystal, viz. quinol-pyridine (1/1), $\mathrm{C}_{6} \mathrm{H}_{6} \mathrm{O}_{2} \cdot \mathrm{C}_{5} \mathrm{H}_{5} \mathrm{~N}$, which does not conform to this expectation. Its stability appears to imply that a combination of individually relatively weak $\mathrm{C}-\mathrm{H} \cdots \mathrm{O}, \mathrm{C}-\mathrm{H} \cdots \pi$ and $\pi-\pi$ stacking interactions are energetically competitive with $\mathrm{O}-\mathrm{H} \cdots \mathrm{N}$ hydrogen bonds. Quinol molecules lie on inversion centres, while pyridine is in a general position.

\section{Comment}

Quinol shows a great propensity for forming co-crystals, and it is widely used to stabilize compounds that are susceptible to polymerization. A search of the Cambridge Structural Database (CSD, Version 5.25; Allen \& Motherwell, 2002) shows that there are 92 co-crystals of quinol with a range of organic compounds. Of all these structures in the CSD, over half contain hydrogen-bond acceptors, e.g. 1,4-dioxane (Barnes et al., 1990). We have recently reported (Oswald et al., 2004) a series of crystal structures of co-crystals of quinol with pyrazine, piperazine, morpholine, pyridine, piperidine and 4,4'-bipyridine (hereafter referred to as guest molecules). These all have closely related packing motifs in which pairs of quinol and guest molecules are connected via $\mathrm{N}-\mathrm{H} \cdots \mathrm{O}$ or $\mathrm{C}-$ $\mathrm{H}$... O interactions.<smiles>Oc1ccc(O)cc1</smiles><smiles>c1ccncc1</smiles>

(I)

Amine $\mathrm{N}$ atoms are more strongly basic than phenol $\mathrm{O}$ atoms and the shortest, and by implication strongest, hydrogen bonds formed in our previous studies were those from a phenol $\mathrm{OH}$ donor to an amine or a pyridine $\mathrm{N}$ atom. Pyrazine and piperazine, which both have two acceptor sites per molecule, were found to form 1:1 co-crystals with quinol, which contains two strong donor functions. In the co-crystals of quinol with molecules with $\mathrm{N}, \mathrm{NH}$ or $\mathrm{NMe}$ and $\mathrm{O}, \mathrm{CH}$ or $\mathrm{CH}_{2}$, respectively in the 1 and 4 positions of a six-membered ring, quinol was found to form hydrogen bonds exclusively to the nitrogen moiety, promoting the formation of quinol-guest co-crystals in a 1:2 ratio. This trend is exemplified by pyridine,
Received 24 August 2004 Accepted 30 September 2004 Online 9 October 2004
(C) 2004 International Union of Crystallography Printed in Great Britain - all rights reserved 


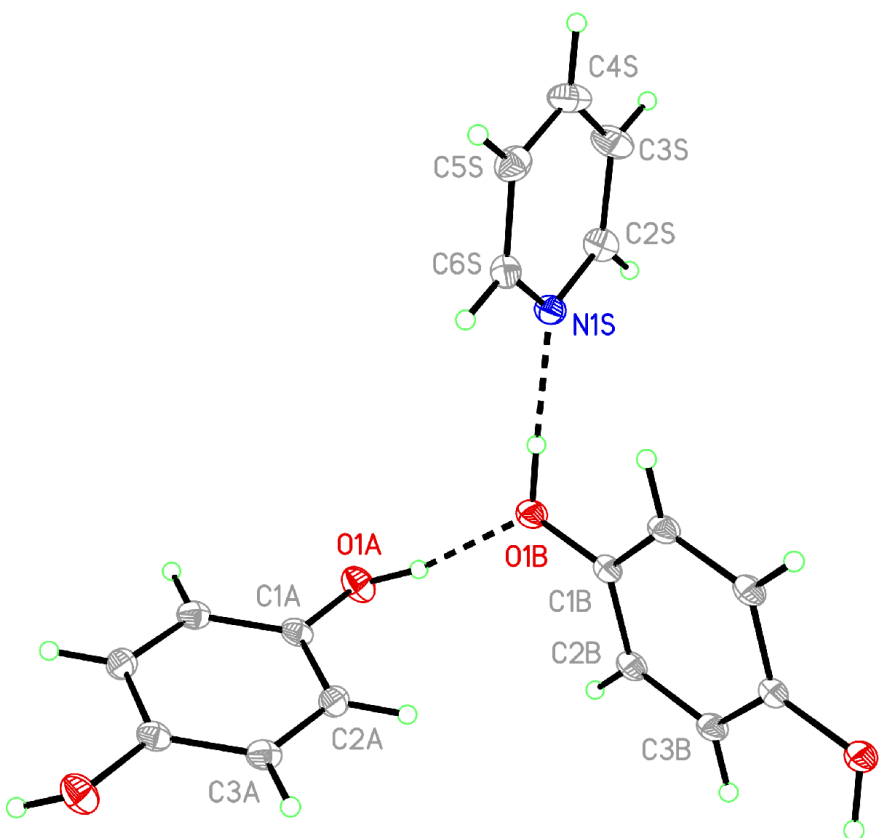

Figure 1

Displacement ellipsoid plot of (I), showing the two crystallographically independent quinol molecules (labelled $A$ and $B$ ) and the pyridine molecule (labelled $S$ ). Ellipsoids are drawn at the $30 \%$ probability level.

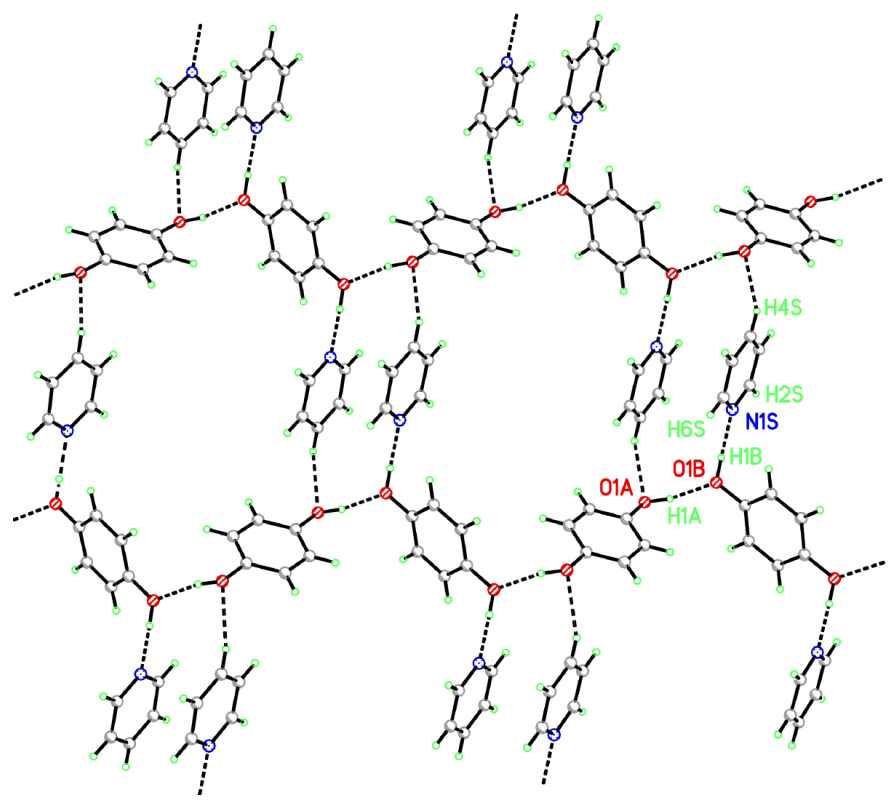

Figure 2

Strong $\mathrm{O}-\mathrm{H} \cdots \mathrm{O}$ hydrogen bonds connect quinol molecules into chains. Pyridine molecules are connected on either side of the chains by $\mathrm{O}-$ $\mathrm{H} \cdots \mathrm{N}$ hydrogen bonds. $\pi-\pi$ Stacking and $\mathrm{C}-\mathrm{H} \cdots \mathrm{O}$ interactions involving the pyridine molecules also link the chains into a layer. The $\mathrm{O}-\mathrm{H} \cdots \mathrm{O}$ and $\mathrm{O}-\mathrm{H} \cdots \mathrm{N}$ hydrogen bonds are shown as heavy dashed lines and the weaker $\mathrm{C}-\mathrm{H} \cdots \mathrm{O}$ interactions as open dashes. This view is along the (112) reciprocal lattice direction.

which forms a co-crystal containing quinol and pyridine in 1:2 ratio.

In this paper, we report the crystal structure of a 1:1 cocrystal of quinol and pyridine, (I), which is an exception to the general stoichiometry rules described above. It was obtained

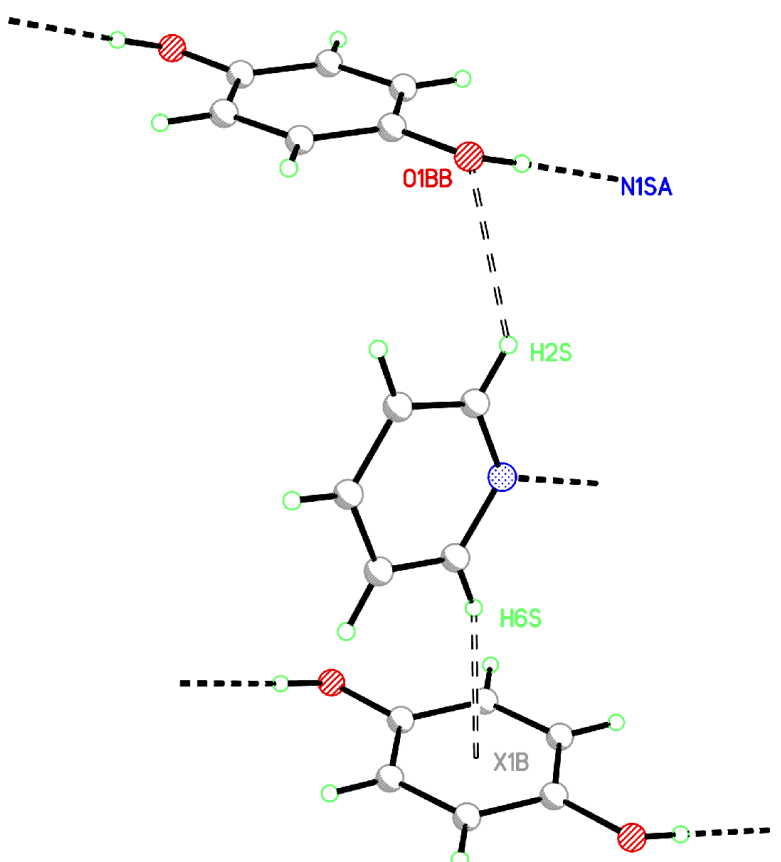

Figure 3

The layers (see Fig. 2) are connected through $\mathrm{C}-\mathrm{H} \cdots \pi$ and $\mathrm{C}-\mathrm{H} \cdots \mathrm{O}$ interactions to the layers above and below. $X 1 B$ is the centroid of the benzene ring (see Table 1). This view is along [010].

by refluxing and then cooling a solution of quinol dissolved in a minimum quantity of pyridine.

Co-crystal (I) crystallizes in space group $P \overline{1}$ and the asymmetric unit consists of one molecule of pyridine in a general position and two half-molecules of quinol (labelled $A$ and $B$ ) residing on crystallographic inversion centres (Fig. 1). Primary bond distances and angles are normal and are listed in the deposited CIF.

The shortest and strongest intermolecular interactions in the structure are $\mathrm{O}-\mathrm{H} \cdots \mathrm{O}$ and $\mathrm{O}-\mathrm{H} \cdots \mathrm{N}$ hydrogen bonds (Fig. 2 and Table 1). Quinol molecules $A$ and $B$ alternate along a chain built by $\mathrm{O}-\mathrm{H} \cdots \mathrm{O}$ hydrogen bonds, in which molecule $A$ provides the $\mathrm{OH}$-donor functions, while molecule $B$ acts as the acceptor. The chains run along the [110] direction and form a $C_{2}^{2}(14)$ graph set (Bernstein et al., 1995). Molecule $B$ donates to pairs of pyridine molecules, which lie on either side of the chain. Successive pyridine molecules are related by the $\overline{1}$ operations centred in the middle of the quinol rings. Cocrystal (I) is the only co-crystal in our study that we have observed in which quinol molecules are directly hydrogen bonded to one another.

The only conventional hydrogen-bonding function 'unsatisfied' by the $\mathrm{N}-\mathrm{H} \cdots \mathrm{O}$ and $\mathrm{O}-\mathrm{H} \cdots \mathrm{O}$ hydrogen bonding described above is the phenol $\mathrm{O}$ atom of molecule $\mathrm{A}$. There is a close contact between this phenol group and a pyridine attached to an adjacent chain $(\mathrm{C} 4 \mathrm{~S}-\mathrm{H} 4 \mathrm{~S} \cdots \mathrm{O} 1 \mathrm{~A}=2.57 \AA$ and $127^{\circ}$ ).

Inter-chain connections are also formed by $\pi-\pi$ stacking between pairs of pyridine molecules. The distance between the atoms in one ring and the mean plane of the other varies in the range 3.532-3.538 (2) $\AA$; the planes are exactly parallel by 
symmetry. The symmetry code relating the rings in this interaction is $(2-x, 1-y,-z)$.

The $\mathrm{C} 4 \mathrm{~S}-\mathrm{H} 4 \mathrm{~S} \cdots \mathrm{O} 1 A$ and $\pi-\pi$ stacking interactions connect the chains into a layer parallel to $(\overline{112})$. The layers are stacked, with quinol molecules above and below the rather 'open' region between the pairs of $\pi$-stacked pyridine rings in Fig. 2. The layers are connected in this region by $\mathrm{C} 6 \mathrm{~S}-$ $\mathrm{H} 6 \mathrm{~S} \cdots \pi$ and $\mathrm{C} 2 \mathrm{~S}-\mathrm{H} 2 \mathrm{~S} \cdots \mathrm{O} 1 B$ interactions, where the $\pi$ acceptor is the aromatic ring from a quinol (molecule $\mathrm{B}$, see Fig. 3). The distance between $\mathrm{H} 6 \mathrm{~S}$ and the centroid of the benzene ring $(X 1 B)$ in this interaction is $2.53 \AA$, with an angle of $162^{\circ}$ subtended at the $\mathrm{H}$ atom. The weak $\mathrm{C} 2 \mathrm{~S}-\mathrm{H} 2 S \ldots \mathrm{O} 1 B$ interaction measures $2.69 \AA$, with an angle at $\mathrm{H} 2 \mathrm{~S}$ of $136^{\circ}$.

$\mathrm{C}-\mathrm{H}$... O hydrogen bonding is now widely accepted (Desiraju \& Steiner, 1999), and weak hydrogen bonding can be exploited in supramolecular chemistry and crystal structure design. For example, $\mathrm{C}-\mathrm{H} \cdots \mathrm{O}$ bonds may play a very important role in protein folding (Derewenda et al., 1995). The $\mathrm{CH}$ groups in pyridine rings are often observed to act as donor groups in $\mathrm{CH}$ - acceptor interactions. Related interactions also occur in heterocyclic compounds related to pyridine, for example, in quinol-pyrazine (Oswald et al., 2004). Such interactions are strong enough that they can be used in crystal engineering, as demonstrated, for example, by Bond (2003) in a series of co-crystals of pyrazine with carboxylic acids. $\pi-\pi$ Stacking has also been observed to be competitive with conventional hydrogen bonding in, for example, the 1:2 cocrystal of quinol with 4,4'-bipyridine (Oswald et al., 2004).

That a co-crystal with 1:1 stoichiometry should be obtained from a mixture of quinol and pyridine must imply that the combination of $\mathrm{C}-\mathrm{H} \cdots \mathrm{O}, \pi-\pi$ stacking and $\mathrm{C}-\mathrm{H} \cdots \pi$ interactions is competitive with $\mathrm{O}-\mathrm{H} \cdots \mathrm{N}$ hydrogen bonding, even for a relatively basic centre such as pyridine.

\section{Experimental}

Starting materials were obtained from Sigma-Aldrich and were used as received. Quinol $(0.49 \mathrm{~g}, 4.45 \mathrm{mmol})$ was refluxed in a minimum volume of pyridine to dissolve the solid. The solution was allowed to cool to room temperature to produce colourless blocks.

\section{Crystal data \\ $\mathrm{C}_{6} \mathrm{H}_{6} \mathrm{O}_{2} \cdot \mathrm{C}_{5} \mathrm{H}_{5} \mathrm{~N}$ \\ $M_{r}=189.21$ \\ Triclinic, $P \overline{1}$ \\ $a=5.7451(5) \AA$ \\ $b=9.1570(9) \AA$ \\ $c=9.6247(9) \AA$ \\ $\alpha=89.002(2)^{\circ}$ \\ $\beta=76.222(2)^{\circ}$ \\ $\gamma=77.478(2)^{\circ}$ \\ $V=479.76(8) \AA^{3}$}

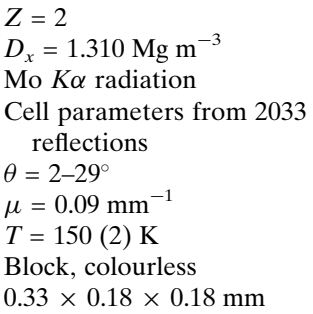

Data collection

Bruker SMART APEX CCD area-

detector diffractometer with

Cryostream cooler (Cosier \& Glazer, 1986)

$\omega$ scans

Absorption correction: multi-scan

(SADABS; Sheldrick, 2004)

$T_{\min }=0.841, T_{\max }=0.980$

2248 independent reflections

1853 reflections with $I>2 \sigma(I)$

$R_{\text {int }}=0.018$

$\theta_{\text {max }}=28.7^{\circ}$

$h=-7 \rightarrow 7$

$k=-12 \rightarrow 12$

$l=-12 \rightarrow 13$

\section{Refinement}

Refinement on $F^{2}$

$R\left[F^{2}>2 \sigma\left(F^{2}\right)\right]=0.048$

$w R\left(F^{2}\right)=0.120$

$S=1.04$

2248 reflections

135 parameters

$\mathrm{H}$ atoms treated by a mixture of independent and constrained refinement

Table 1

Hydrogen-bonding geometry $\left(\AA{ }^{\circ}{ }^{\circ}\right)$.

\begin{tabular}{lllll}
\hline$D-\mathrm{H} \cdots A$ & $D-\mathrm{H}$ & $\mathrm{H} \cdots A$ & $D \cdots A$ & $D-\mathrm{H} \cdots A$ \\
\hline $\mathrm{O} 1 A-\mathrm{H} 1 A \cdots \mathrm{O} 1 B^{\mathrm{i}}$ & $0.94(3)$ & $1.81(3)$ & $2.7392(16)$ & $168(2)$ \\
$\mathrm{O} 1 B-\mathrm{H} 1 B \cdots \mathrm{N} 1 S^{\mathrm{ii}}$ & $0.93(2)$ & $1.79(2)$ & $2.7178(17)$ & $178(2)$ \\
$\mathrm{C} 2 \mathrm{~S}-\mathrm{H} 2 S \cdots \mathrm{O} 1 B$ & 0.95 & 2.69 & $3.436(2)$ & 136 \\
$\mathrm{C} 4 \mathrm{~S}-\mathrm{H} 4 S \cdots \mathrm{O} 1 A$ & 0.95 & 2.57 & $3.225(2)$ & 127 \\
$\mathrm{C} 6 \mathrm{~S}-\mathrm{H} 6 S \cdots X 1 B^{\mathrm{iii}}$ & 0.95 & 2.53 & 3.45 & 162 \\
\hline
\end{tabular}

Symmetry codes: (i) $1+x, y, z$; (ii) $1-x, 1-y,-z$; (iii) $1+x, y, z-1 . X 1 B$ is the centroid of the benzene ring.

$\mathrm{H}$ atoms were placed on $\mathrm{C}$ atoms in calculated positions $(\mathrm{C}-\mathrm{O}=$ $0.95 \AA$ ) and allowed to ride on their parent atoms $\left[U_{\text {iso }}(\mathrm{H})=\right.$ $\left.1.2 U_{\text {eq }}(\mathrm{C})\right]$. Hydroxyl $\mathrm{H}$ atoms were located in difference maps and refined freely.

Data collection: SMART (Bruker-Nonius, 2001); cell refinement: SAINT (Bruker-Nonius, 2003); data reduction: SAINT; program(s) used to solve structure: SHELXTL (Sheldrick, 2001); program(s) used to refine structure: SHELXTL; molecular graphics: SHELXTL; software used to prepare material for publication: SHELXTL, PLATON [Spek (2004) as incorporated in WinGX (Farrugia, 1999)] and enCIFer (Version 1.1; Allen et al., 2004).

The authors thank the EPSRC, The University of Edinburgh and The Cambridge Crystallographic Data Centre for funding.

\section{References}

Allen, F. H., Johnson, O., Shields, G. P., Smith, B. R. \& Towler, M. (2004). J. Appl. Cryst. 37, 335-338.

Allen, F. H. \& Motherwell, W. D. S. (2002). Acta Cryst. B58, 407-422.

Barnes, J. C., Paton, J. D. \& Blyth, C. S. (1990). Acta Cryst. C46, 1183-1184.

Bernstein, J., Davis, R. E., Shimoni, L. \& Chang, N.-L. (1995). Angew. Chem. Int. Ed. Engl. 34, 1555-1573.

Bond, A. D. (2003). Chem. Commun. pp. 250-251.

Bruker-Nonius (2001). SMART. Version 5.624. Bruker-Nonius Inc., Madison, Wisconsin, USA.

Bruker-Nonius (2003). SAINT. Version 7. Bruker-Nonius Inc., Madison, Wisconsin, USA.

Cosier, J. \& Glazer, A. M. (1986). J. Appl. Cryst. 19, 105-107.

Derewenda, Z. S., Lee, L. \& Derewenda, U. (1995). J. Mol. Biol. 252, 248-262.

Desiraju, G. R. \& Steiner, T. (1999). The Weak Hydrogen Bond. IUCr Monographs on Crystallography, No. 9. Oxford University Press.

Farrugia, L. J. (1999). J. Appl. Cryst. 32, 837-838.

Oswald, I. D. H., Motherwell, W. D. S. \& Parsons, S. (2004). Acta Cryst. B60. Submitted.

Sheldrick, G. M. (2001). SHELXTL. Version 6.01. Bruker-Nonius Inc., Madison, Wisconsin, USA.

Sheldrick, G. M. (2004). SADABS. University of Göttingen, Germany.

Spek, A. L. (2004). PLATON. Utrecht University, The Netherlands. 


\title{
supporting information
}

Acta Cryst. (2004). E60, o1967-o1969 [https://doi.org/10.1107/S1600536804024547]

\section{A 1:1 co-crystal of quinol and pyridine}

\author{
Iain D. H. Oswald, W. D. Sam Motherwell and Simon Parsons
}

Quinol-Pyridine (1/1)

Crystal data

$\mathrm{C}_{6} \mathrm{H}_{6} \mathrm{O}_{2} \cdot \mathrm{C}_{5} \mathrm{H}_{5} \mathrm{~N}$

$Z=2$

$M_{r}=189.21$

Triclinic, $P \overline{1}$

Hall symbol: -P1

$a=5.7451(5) \AA$

$b=9.1570(9) \AA$

$c=9.6247(9) \AA$

$\alpha=89.002(2)^{\circ}$

$\beta=76.222(2)^{\circ}$

$\gamma=77.478(2)^{\circ}$

$V=479.76(8) \AA^{3}$

$F(000)=200$

$D_{\mathrm{x}}=1.310 \mathrm{Mg} \mathrm{m}^{-3}$

Mo $K \alpha$ radiation, $\lambda=0.71073 \AA$

Cell parameters from 2033 reflections

$\theta=2-29^{\circ}$

$\mu=0.09 \mathrm{~mm}^{-1}$

$T=150 \mathrm{~K}$

Block, colourless

$0.33 \times 0.18 \times 0.18 \mathrm{~mm}$

\section{Data collection}

Bruker APEX CCD area-detector diffractometer

Radiation source: fine-focus sealed tube

4349 measured reflections

2248 independent reflections

Graphite monochromator

$\omega$ scans

Absorption correction: multi-scan

1853 reflections with $I>2 \Sigma(I)$

$R_{\text {int }}=0.018$

$\theta_{\max }=28.7^{\circ}, \theta_{\min }=2.2^{\circ}$

(SADABS; Sheldrick, 2004)

$T_{\min }=0.841, T_{\max }=0.980$

$h=-7 \rightarrow 7$

$k=-12 \rightarrow 12$

$l=-12 \rightarrow 13$

\section{Refinement}

Refinement on $F^{2}$

Least-squares matrix: full

$R\left[F^{2}>2 \sigma\left(F^{2}\right)\right]=0.048$

$w R\left(F^{2}\right)=0.120$

$S=1.04$

2248 reflections

135 parameters

0 restraints

Primary atom site location: structure-invariant

direct methods

Secondary atom site location: difference Fourier map

Hydrogen site location: geom and difmap $(\mathrm{OH})$

$\mathrm{H}$ atoms treated by a mixture of independent and constrained refinement

$w=1 /\left[\sigma^{2}\left(F_{0}^{2}\right)+(0.049 P)^{2}+0.1585 P\right]$

where $P=\left(F_{\mathrm{o}}{ }^{2}+2 F_{\mathrm{c}}{ }^{2}\right) / 3$

$(\Delta / \sigma)_{\max }=0.001$

$\Delta \rho_{\max }=0.28 \mathrm{e} \AA^{-3}$

$\Delta \rho_{\min }=-0.21$ e $\AA^{-3}$

Special details

Geometry. All s.u.s are estimated using the full covariance matrix. The cell e.s.d.'s are taken into account individually in the estimation of e.s.d.'s in distances, angles and torsion angles; correlations between e.s.d.'s in cell parameters are only used when they are defined by crystal symmetry. S.u.s are not quoted for interactions involving calculated $\mathrm{H}$-atom positions. 
Refinement. 912_ALERT_3_B Missing FCF Reflections Above STH $/ L=0.6 \cdots \cdots . .228$ 911_ALERT_3_C Missing FCF Refl. Between $\mathrm{TH}(\mathrm{Min}) \& \overline{\mathrm{ST}} \mathrm{H} / L=0.6 . .8$

Not serious. Coverage statistics:

Resolution \& Completeness Statistics (Cumulative)

$\sin ($ th)/Lambda Complete Expected Measured Missing

20.820 .5001 .00010041004023 .010 .5500 .99813341331325 .240 .6000 .995173417268

ACTA Min. Res. —- 27.510 .6500 .975220821525629 .840 .7000 .90524842248236

061_ALERT_3_C Tmax/Tmin Range Test $R R^{\prime}$ too Large …….... 0.87

Tmin and Tmax reported: $0.8410 .980 \mathrm{Tmin}^{\prime}$ and Tmax expected: 0.9700 .984

Noted, but no action taken. $S A D A B S$ attempts to correct for all systematic errors not just absorption. The large range could represent a small amount of crystal decay for example.

480_ALERT_4_C Long H“..A H-Bond Reported H2S.. O1B.. 2.69 A ng.

See text.

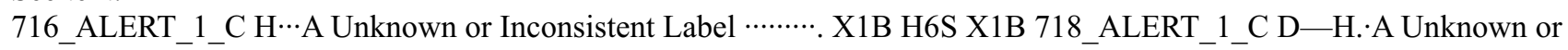
Inconsistent Label …… X1B C6S H6S X1B

See Table 1 and Figure 3.

764_ALERT_4_C Overcomplete CIF Bond List Detected (Rep/Expd). 1.14 Ratio

Noted; no action taken. The quinol molecules are on -1 sites, which may explain why this has happened.

Fractional atomic coordinates and isotropic or equivalent isotropic displacement parameters $\left(\AA^{2}\right)$

\begin{tabular}{|c|c|c|c|c|}
\hline & $x$ & $y$ & $z$ & $U_{\text {iso }} * / U_{\text {eq }}$ \\
\hline O1A & $1.3232(2)$ & $0.07757(13)$ & $0.26274(12)$ & $0.0362(3)$ \\
\hline H1A & $1.344(4)$ & $0.174(3)$ & $0.281(2)$ & $0.065(7)^{*}$ \\
\hline $\mathrm{C} 1 \mathrm{~A}$ & $1.1638(3)$ & $0.04088(16)$ & $0.38221(16)$ & $0.0263(3)$ \\
\hline $\mathrm{C} 2 \mathrm{~A}$ & $1.1609(3)$ & $0.08591(16)$ & $0.51998(17)$ & $0.0278(3)$ \\
\hline $\mathrm{H} 2 \mathrm{~A}$ & 1.2717 & 0.1445 & 0.5340 & $0.033^{*}$ \\
\hline $\mathrm{C} 3 \mathrm{~A}$ & $0.9969(3)$ & $0.04557(16)$ & $0.63687(16)$ & $0.0283(3)$ \\
\hline $\mathrm{H} 3 \mathrm{~A}$ & 0.9948 & 0.0775 & 0.7306 & $0.034 *$ \\
\hline O1B & $0.3575(2)$ & $0.36954(12)$ & $0.28535(12)$ & $0.0290(3)$ \\
\hline H1B & $0.268(4)$ & $0.441(3)$ & $0.238(2)$ & $0.057(6)^{*}$ \\
\hline $\mathrm{C} 1 \mathrm{~B}$ & $0.4247(3)$ & $0.43589(16)$ & $0.39227(15)$ & $0.0239(3)$ \\
\hline $\mathrm{C} 2 \mathrm{~B}$ & $0.6167(3)$ & $0.35608(16)$ & $0.44644(16)$ & $0.0256(3)$ \\
\hline $\mathrm{H} 2 \mathrm{~B}$ & 0.6960 & 0.2573 & 0.4106 & $0.031 *$ \\
\hline $\mathrm{C} 3 \mathrm{~B}$ & $0.6928(3)$ & $0.42029(16)$ & $0.55269(16)$ & $0.0252(3)$ \\
\hline H3B & 0.8258 & 0.3657 & 0.5881 & $0.030 *$ \\
\hline N1S & $0.8953(2)$ & $0.41739(14)$ & $-0.14401(14)$ & $0.0283(3)$ \\
\hline $\mathrm{C} 2 \mathrm{~S}$ & $0.8062(3)$ & $0.34857(18)$ & $-0.02635(17)$ & $0.0330(4)$ \\
\hline $\mathrm{H} 2 \mathrm{~S}$ & 0.6370 & 0.3800 & 0.0198 & $0.040^{*}$ \\
\hline $\mathrm{C} 3 \mathrm{~S}$ & $0.9484(4)$ & $0.2340(2)$ & $0.03146(18)$ & $0.0408(4)$ \\
\hline $\mathrm{H} 3 \mathrm{~S}$ & 0.8787 & 0.1883 & 0.1160 & $0.049 *$ \\
\hline $\mathrm{C} 4 \mathrm{~S}$ & $1.1934(4)$ & $0.1870(2)$ & -0.03559 (19) & $0.0398(4)$ \\
\hline $\mathrm{H} 4 \mathrm{~S}$ & 1.2952 & 0.1076 & 0.0013 & $0.048^{*}$ \\
\hline C5S & $1.2883(3)$ & 0.25698 (19) & $-0.15699(18)$ & $0.0348(4)$ \\
\hline H5S & 1.4567 & 0.2272 & -0.2052 & $0.042 *$ \\
\hline C6S & $1.1336(3)$ & $0.37130(18)$ & $-0.20724(17)$ & $0.0300(3)$ \\
\hline H6S & 1.1997 & 0.4196 & -0.2909 & $0.036^{*}$ \\
\hline
\end{tabular}


Atomic displacement parameters $\left(\AA^{2}\right)$

\begin{tabular}{lllllll}
\hline & $U^{11}$ & $U^{22}$ & $U^{33}$ & $U^{12}$ & $U^{13}$ & $U^{23}$ \\
\hline O1A & $0.0467(7)$ & $0.0270(6)$ & $0.0325(6)$ & $-0.0101(5)$ & $-0.0030(5)$ & $0.0004(5)$ \\
C1A & $0.0313(8)$ & $0.0171(6)$ & $0.0285(8)$ & $-0.0004(6)$ & $-0.0075(6)$ & $0.0016(5)$ \\
C2A & $0.0311(8)$ & $0.0199(7)$ & $0.0341(8)$ & $-0.0040(6)$ & $-0.0123(6)$ & $-0.0008(6)$ \\
C3A & $0.0379(8)$ & $0.0202(7)$ & $0.0267(7)$ & $-0.0015(6)$ & $-0.0118(6)$ & $-0.0013(5)$ \\
O1B & $0.0364(6)$ & $0.0207(5)$ & $0.0317(6)$ & $-0.0040(4)$ & $-0.0136(5)$ & $0.0021(4)$ \\
C1B & $0.0261(7)$ & $0.0197(7)$ & $0.0258(7)$ & $-0.0054(5)$ & $-0.0057(6)$ & $0.0038(5)$ \\
C2B & $0.0269(7)$ & $0.0167(7)$ & $0.0303(8)$ & $-0.0008(5)$ & $-0.0051(6)$ & $0.0021(5)$ \\
C3B & $0.0244(7)$ & $0.0200(7)$ & $0.0296(8)$ & $-0.0011(5)$ & $-0.0070(6)$ & $0.0045(5)$ \\
N1S & $0.0329(7)$ & $0.0242(6)$ & $0.0292(7)$ & $-0.0052(5)$ & $-0.0110(5)$ & $0.0024(5)$ \\
C2S & $0.0351(8)$ & $0.0323(8)$ & $0.0288(8)$ & $-0.0043(7)$ & $-0.0048(7)$ & $0.0001(6)$ \\
C3S & $0.0556(11)$ & $0.0361(9)$ & $0.0266(8)$ & $-0.0049(8)$ & $-0.0072(8)$ & $0.0075(7)$ \\
C4S & $0.0510(11)$ & $0.0316(9)$ & $0.0353(9)$ & $0.0048(8)$ & $-0.0199(8)$ & $0.0011(7)$ \\
C5S & $0.0313(8)$ & $0.0348(9)$ & $0.0377(9)$ & $-0.0026(7)$ & $-0.0111(7)$ & $-0.0049(7)$ \\
C6S & $0.0329(8)$ & $0.0286(8)$ & $0.0317(8)$ & $-0.0111(6)$ & $-0.0098(6)$ & $0.0030(6)$ \\
& & & & & &
\end{tabular}

Geometric parameters $\left(\AA,{ }^{\circ}\right)$

\begin{tabular}{|c|c|c|c|}
\hline $\mathrm{O} 1 \mathrm{~A}-\mathrm{C} 1 \mathrm{~A}$ & $1.3755(19)$ & $\mathrm{C} 3 \mathrm{~B}-\mathrm{C} 1 \mathrm{~B}^{\mathrm{ii}}$ & $1.392(2)$ \\
\hline $\mathrm{O} 1 \mathrm{~A}-\mathrm{H} 1 \mathrm{~A}$ & $0.94(3)$ & $\mathrm{C} 3 \mathrm{~B}-\mathrm{H} 3 \mathrm{~B}$ & 0.9500 \\
\hline $\mathrm{C} 1 \mathrm{~A}-\mathrm{C} 3 \mathrm{~A}^{\mathrm{i}}$ & $1.384(2)$ & $\mathrm{N} 1 \mathrm{~S}-\mathrm{C} 2 \mathrm{~S}$ & $1.334(2)$ \\
\hline $\mathrm{C} 1 \mathrm{~A}-\mathrm{C} 2 \mathrm{~A}$ & $1.391(2)$ & $\mathrm{N} 1 \mathrm{~S}-\mathrm{C} 6 \mathrm{~S}$ & $1.336(2)$ \\
\hline $\mathrm{C} 2 \mathrm{~A}-\mathrm{C} 3 \mathrm{~A}$ & $1.386(2)$ & $\mathrm{C} 2 \mathrm{~S}-\mathrm{C} 3 \mathrm{~S}$ & $1.379(2)$ \\
\hline $\mathrm{C} 2 \mathrm{~A}-\mathrm{H} 2 \mathrm{~A}$ & 0.9500 & $\mathrm{C} 2 \mathrm{~S}-\mathrm{H} 2 \mathrm{~S}$ & 0.9500 \\
\hline $\mathrm{C} 3 \mathrm{~A}-\mathrm{C} 1 \mathrm{~A}^{\mathrm{i}}$ & $1.384(2)$ & $\mathrm{C} 3 \mathrm{~S}-\mathrm{C} 4 \mathrm{~S}$ & $1.377(3)$ \\
\hline $\mathrm{C} 3 \mathrm{~A}-\mathrm{H} 3 \mathrm{~A}$ & 0.9500 & $\mathrm{C} 3 \mathrm{~S}-\mathrm{H} 3 \mathrm{~S}$ & 0.9500 \\
\hline $\mathrm{O} 1 \mathrm{~B}-\mathrm{C} 1 \mathrm{~B}$ & $1.3745(18)$ & $\mathrm{C} 4 \mathrm{~S}-\mathrm{C} 5 \mathrm{~S}$ & $1.377(3)$ \\
\hline $\mathrm{O} 1 \mathrm{~B}-\mathrm{H} 1 \mathrm{~B}$ & $0.93(2)$ & $\mathrm{C} 4 \mathrm{~S}-\mathrm{H} 4 \mathrm{~S}$ & 0.9500 \\
\hline $\mathrm{C} 1 \mathrm{~B}-\mathrm{C} 2 \mathrm{~B}$ & $1.391(2)$ & $\mathrm{C} 5 \mathrm{~S}-\mathrm{C} 6 \mathrm{~S}$ & $1.380(2)$ \\
\hline $\mathrm{C} 1 \mathrm{~B}-\mathrm{C} 3 \mathrm{~B}^{\mathrm{ii}}$ & $1.392(2)$ & $\mathrm{C} 5 \mathrm{~S}-\mathrm{H} 5 \mathrm{~S}$ & 0.9500 \\
\hline $\mathrm{C} 2 \mathrm{~B}-\mathrm{C} 3 \mathrm{~B}$ & $1.389(2)$ & $\mathrm{C} 6 \mathrm{~S}-\mathrm{H} 6 \mathrm{~S}$ & 0.9500 \\
\hline $\mathrm{C} 2 \mathrm{~B}-\mathrm{H} 2 \mathrm{~B}$ & 0.9500 & & \\
\hline $\mathrm{C} 1 \mathrm{~A}-\mathrm{O} 1 \mathrm{~A}-\mathrm{H} 1 \mathrm{~A}$ & $107.2(14)$ & $\mathrm{C} 2 \mathrm{~B}-\mathrm{C} 3 \mathrm{~B}-\mathrm{H} 3 \mathrm{~B}$ & 119.8 \\
\hline $\mathrm{O} 1 \mathrm{~A}-\mathrm{C} 1 \mathrm{~A}-\mathrm{C} 3 \mathrm{~A}^{\mathrm{i}}$ & $118.11(14)$ & $\mathrm{C} 1 \mathrm{~B}^{\mathrm{ii}}-\mathrm{C} 3 \mathrm{~B}-\mathrm{H} 3 \mathrm{~B}$ & 119.8 \\
\hline $\mathrm{O} 1 \mathrm{~A}-\mathrm{C} 1 \mathrm{~A}-\mathrm{C} 2 \mathrm{~A}$ & $122.49(14)$ & $\mathrm{C} 2 \mathrm{~S}-\mathrm{N} 1 \mathrm{~S}-\mathrm{C} 6 \mathrm{~S}$ & $117.48(14)$ \\
\hline $\mathrm{C} 3 \mathrm{~A}^{\mathrm{i}}-\mathrm{C} 1 \mathrm{~A}-\mathrm{C} 2 \mathrm{~A}$ & $119.40(14)$ & $\mathrm{N} 1 \mathrm{~S}-\mathrm{C} 2 \mathrm{~S}-\mathrm{C} 3 \mathrm{~S}$ & $123.05(16)$ \\
\hline $\mathrm{C} 3 \mathrm{~A}-\mathrm{C} 2 \mathrm{~A}-\mathrm{C} 1 \mathrm{~A}$ & $120.20(14)$ & $\mathrm{N} 1 \mathrm{~S}-\mathrm{C} 2 \mathrm{~S}-\mathrm{H} 2 \mathrm{~S}$ & 118.5 \\
\hline $\mathrm{C} 3 \mathrm{~A}-\mathrm{C} 2 \mathrm{~A}-\mathrm{H} 2 \mathrm{~A}$ & 119.9 & $\mathrm{C} 3 \mathrm{~S}-\mathrm{C} 2 \mathrm{~S}-\mathrm{H} 2 \mathrm{~S}$ & 118.5 \\
\hline $\mathrm{C} 1 \mathrm{~A}-\mathrm{C} 2 \mathrm{~A}-\mathrm{H} 2 \mathrm{~A}$ & 119.9 & $\mathrm{C} 4 \mathrm{~S}-\mathrm{C} 3 \mathrm{~S}-\mathrm{C} 2 \mathrm{~S}$ & $118.77(16)$ \\
\hline $\mathrm{C} 1 \mathrm{~A}-\mathrm{C} 3 \mathrm{~A}-\mathrm{C} 2 \mathrm{~A}$ & $120.40(14)$ & $\mathrm{C} 4 \mathrm{~S}-\mathrm{C} 3 \mathrm{~S}-\mathrm{H} 3 \mathrm{~S}$ & 120.6 \\
\hline $\mathrm{C} 1 \mathrm{~A}^{\mathrm{i}}-\mathrm{C} 3 \mathrm{~A}-\mathrm{H} 3 \mathrm{~A}$ & 119.8 & $\mathrm{C} 2 \mathrm{~S}-\mathrm{C} 3 \mathrm{~S}-\mathrm{H} 3 \mathrm{~S}$ & 120.6 \\
\hline $\mathrm{C} 2 \mathrm{~A}-\mathrm{C} 3 \mathrm{~A}-\mathrm{H} 3 \mathrm{~A}$ & 119.8 & $\mathrm{C} 5 \mathrm{~S}-\mathrm{C} 4 \mathrm{~S}-\mathrm{C} 3 \mathrm{~S}$ & $118.90(16)$ \\
\hline $\mathrm{C} 1 \mathrm{~B}-\mathrm{O} 1 \mathrm{~B}-\mathrm{H} 1 \mathrm{~B}$ & $110.5(14)$ & $\mathrm{C} 5 \mathrm{~S}-\mathrm{C} 4 \mathrm{~S}-\mathrm{H} 4 \mathrm{~S}$ & 120.6 \\
\hline $\mathrm{O} 1 \mathrm{~B}-\mathrm{C} 1 \mathrm{~B}-\mathrm{C} 2 \mathrm{~B}$ & $118.52(13)$ & $\mathrm{C} 3 \mathrm{~S}-\mathrm{C} 4 \mathrm{~S}-\mathrm{H} 4 \mathrm{~S}$ & 120.6 \\
\hline $\mathrm{O} 1 \mathrm{~B}-\mathrm{C} 1 \mathrm{~B}-\mathrm{C} 3 \mathrm{~B}^{\mathrm{ii}}$ & $122.17(13)$ & $\mathrm{C} 4 \mathrm{~S}-\mathrm{C} 5 \mathrm{~S}-\mathrm{C} 6 \mathrm{~S}$ & $118.62(16)$ \\
\hline
\end{tabular}


supporting information

$\begin{array}{ll}\mathrm{C} 2 \mathrm{~B}-\mathrm{C} 1 \mathrm{~B}-\mathrm{C} 3 \mathrm{~B}^{\mathrm{ii}} & 119.31(14) \\ \mathrm{C} 3 \mathrm{~B}-\mathrm{C} 2 \mathrm{~B}-\mathrm{C} 1 \mathrm{~B} & 120.23(13) \\ \mathrm{C} 3 \mathrm{~B}-\mathrm{C} 2 \mathrm{~B}-\mathrm{H} 2 \mathrm{~B} & 119.9 \\ \mathrm{C} 1 \mathrm{~B}-\mathrm{C} 2 \mathrm{~B}-\mathrm{H} 2 \mathrm{~B} & 119.9 \\ \mathrm{C} 2 \mathrm{~B}-\mathrm{C} 3 \mathrm{~B}-\mathrm{C} 1 \mathrm{~B}^{\mathrm{ii}} & 120.45(13)\end{array}$

$\mathrm{C} 4 \mathrm{~S}-\mathrm{C} 5 \mathrm{~S}-\mathrm{H} 5 \mathrm{~S}$

120.7

$\mathrm{C} 6 \mathrm{~S}-\mathrm{C} 5 \mathrm{~S}-\mathrm{H} 5 \mathrm{~S}$

120.7

$\mathrm{N} 1 \mathrm{~S}-\mathrm{C} 6 \mathrm{~S}-\mathrm{C} 5 \mathrm{~S}$

$123.18(15)$

$\mathrm{N} 1 \mathrm{~S}-\mathrm{C} 6 \mathrm{~S}-\mathrm{H} 6 \mathrm{~S}$

118.4

$\mathrm{C} 5 \mathrm{~S}-\mathrm{C} 6 \mathrm{~S}-\mathrm{H} 6 \mathrm{~S}$

118.4

Symmetry codes: (i) $-x+2,-y,-z+1$; (ii) $-x+1,-y+1,-z+1$.

Hydrogen-bond geometry $\left(\AA,{ }^{\circ}\right)$

\begin{tabular}{lllll}
\hline$D-\mathrm{H} \cdots A$ & $D-\mathrm{H}$ & $\mathrm{H} \cdots A$ & $D \cdots A$ & $D-\mathrm{H} \cdots A$ \\
\hline $\mathrm{O} 1 A-\mathrm{H} 1 A \cdots \mathrm{O} 1 B^{\mathrm{iii}}$ & $0.94(3)$ & $1.81(3)$ & $2.7392(16)$ & $168(2)$ \\
$\mathrm{O} 1 B-\mathrm{H} 1 B \cdots \mathrm{N} 1 S^{\mathrm{iv}}$ & $0.93(2)$ & $1.79(2)$ & $2.7178(17)$ & $178(2)$ \\
$\mathrm{C} 2 S-\mathrm{H} 2 S \cdots \mathrm{O} 1 B$ & 0.95 & 2.69 & $3.436(2)$ & 136 \\
$\mathrm{C} 4 S-\mathrm{H} 4 S \cdots \mathrm{O} 1 A$ & 0.95 & 2.57 & $3.225(2)$ & 127 \\
$\mathrm{C} 6 S-\mathrm{H} 6 S \cdots \mathrm{X} 1 B^{\mathrm{v}}$ & 0.95 & 2.53 & 3.45 & 162
\end{tabular}

Symmetry codes: (iii) $x+1, y, z$; (iv) $-x+1,-y+1,-z$; (v) $x+1, y, z-1$. 